\title{
Utilization of peripherin and S-100 immunohistochemistry in the diagnosis of Hirschsprung disease
}

\author{
Susan K Holland ${ }^{1}$, Richard B Hessler ${ }^{2}$, Michelle D Reid-Nicholson ${ }^{1}$, Preetha Ramalingam ${ }^{1}$ \\ and Jeffrey R Lee ${ }^{3}$ \\ ${ }^{1}$ Department of Pathology, Medical College of Georgia, Augusta, GA, USA; ${ }^{2}$ Department of Pathology, \\ Erhanger Health System, Chattanooga, TN, USA and ${ }^{3}$ Department of Pathology, Immunotherapy Center, and \\ Institute of Molecular Medicine and Genetics, Medical College of Georgia, and Charlie Norwood Veterans \\ Affairs Medical Center, Augusta, GA, USA
}

\begin{abstract}
Evaluation of rectal biopsies for ganglion cells is performed for patients suspected of having Hirschsprung disease. At times, identification of ganglion cells can be difficult, especially in newborns. To assist in diagnosis, frozen tissue can be collected for acetylcholinesterase histochemical staining. At our institution, we developed a protocol using peripherin and S-100 immunostaining as an adjunct to hematoxylin and eosin (H\&E) for the identification of ganglion cells. Further, at the time of frozen section, we performed Diff Quik staining to highlight ganglion cells. One hundred and thirty eight rectal biopsies submitted for evaluation of Hirschsprung disease were compiled from the archives of the Medical College of Georgia from 2002 to 2009. Initial evaluation consisted of eight levels of H\&E-stained slides and two unstained slides each for immunostaining with peripherin and S-100. If on initial evaluation, ganglion cells were not identified, additional H\&E and peripherin immunostains were performed. Peripherin immunostaining was unequivocally identified in the cytoplasm of ganglion cells of patients at all ages. Of the 136 patients with diagnostic biopsies, $80 \%$ had ganglion cells. Of these, $93 \%$ of cases were diagnosed on the original eight levels. Twenty-seven cases were devoid of ganglion cells, and of these, $81 \%$ showed submucosal neural hypertrophy on S-100 staining. Twenty-six patients had confirmed aganglionic segments at the time of colonic resection. One patient had colostomy only. A total of 54 frozen sections were performed on 25 patients over this same period of time. Diff Quick staining was found to be very useful. In this study, our protocol proved to be very sensitive, specific, and efficient for the diagnosis of Hirschsprung disease.
\end{abstract}

Modern Pathology (2010) 23, 1173-1179; doi:10.1038/modpathol.2010.104; published online 21 May 2010

Keywords: ganglion cells; Hirschsprung disease; peripherin

Congenital intestinal aganglionosis, or Hirschsprung disease, results from a malformation of the enteric nervous system. Adequate motility of the gastrointestinal tract requires innervation by a properly developed and mature enteric nervous system. The absence of intrinsic ganglion cells from the distal rectum and a variable length of the contiguous

Correspondence: Dr JR Lee, MD, Department of Pathology, Charlie Norwood Veterans Affairs Medical Center, One Freedom Way, Augusta, Georgia 30904, USA.

E-mail: jlee@mcg.edu

This work was presented in part at the 98th annual meeting of the United States and Canadian Academy of Pathology, 7-13 March 2009, Boston, MA, USA.

Received 25 November 2009; revised 29 March 2010; accepted 30 March 2010; published online 21 May 2010 bowel is the required diagnostic feature of Hirschsprung disease. In embryologic development, enteric nerve fibers migrate caudally with vagal fibers and arrive in the colon by 12 weeks. Over a decade ago, loss of function mutations of multiple genes, including the ret proto-oncogene, were found to be involved in the development of aganglionosis. ${ }^{1}$ It is now known that not only do these 'loss of function' mutations affect development and migration of ganglion cells, but they are also implicated in early ganglion cell death. ${ }^{2}$

Evaluation of rectal biopsies for the presence or absence of ganglion cells is performed in patients suspected of having Hirschsprung disease. At times, the identification of ganglion cells can be difficult, especially in the population at greatest risk of 
presenting with Hirschsprung disease, the newborn. In these patients, immature ganglion cells can be confused with endothelial cells, other mesenchymal cells, and inflammatory cells. For this reason, many institutions use acetylcholinesterase histochemical staining on frozen rectal tissue as an adjunct to the diagnosis of aganglionosis. However, the accurate diagnosis in these frozen section stains requires a high level of expertise, especially in equivocal cases. $^{3,4}$ As an alternate, identification of neural hypertrophy can be a useful adjunctive diagnostic modality in cases of aganglionosis. It has been reported that $90 \%$ of rectal biopsies in patients with Hirschsprung disease show submucosal nerve trunks with a diameter of $\geqslant 40 \mu \mathrm{m}$. $^{5}$

As a result of these diagnostic difficulties, there have been a number of immunohistochemical stains developed to help identify ganglion cells. Antibodies against neuron-specific enolase (NSE), ${ }^{6,7}$ peripherin, ${ }^{7-10}$ cathepsin $\mathrm{D},{ }^{8,11} \mathrm{bcl}-2,{ }^{12} \mathrm{NeuN},{ }^{13}$ bone morphogenetic protein receptor IA (BMPRIA), ${ }^{14}$ neurofilament, ${ }^{7,15}$ tubulin, ${ }^{15}$ ret oncoprotein, ${ }^{16}$ and recently, MAP- $2^{17}$ have all been successful at highlighting ganglion cells that are difficult to identify on hematoxylin and eosin ( $\mathrm{H} \& \mathrm{E})$ staining.

In 2002, we developed and implemented a protocol using peripherin immunohistochemical staining, coupled with H\&E evaluations, in the identification of ganglion cells in rectal biopsies. After reviewing the literature, we selected peripherin to highlight the ganglion cells, as it showed robust staining of ganglion cells in the adult and pediatric intestinal tissues. We did note that there was weak granular nerve fiber immunostaining; however, it was never difficult to differentiate this staining from the dense typical cytoplasmic staining of single and clusters of ganglion cells. We also performed S-100 immunohistochemical staining as an adjunct to identifying neural hypertrophy. Herein, are the results of our protocol over the past 7 years.

\section{Materials and methods}

\section{Protocol}

The protocol consists of eight H\&E levels, two peripherin, and two S-100 immunostains. The unstained slides for immunostains were obtained at levels 3, 4 and 8, 9 in the block. If ganglion cells were not identified with the initial protocol, then additional $\mathrm{H} \& \mathrm{E}$ and peripherin stains were performed.

Over the 7-year period of study, 10 pathologists from our institution have interpreted these rectal biopsies. If there was an absence of ganglion cells on the initial protocol, there was variation between diagnosing pathologists on the number of additional H\&E levels and immunostains that would be ordered. The number of H\&Es ordered ranged from 10 additional levels to serial sections through the block.

\section{Case Evaluations and Follow-Up}

After Institutional Review Board approval, all biopsies obtained to rule out Hirschsprung disease were retrieved from the pathology archives of the Medical College of Georgia from 2002 to 2009. All cases were diagnosed with the assistance of the peripherin and S-100 immunohistochemical-staining protocol. Each case was evaluated for the presence or absence of ganglion cells and neural hypertrophy. If ganglion cells were present, we determined whether they were discovered on initial protocol or on repeat levels. If ganglion cells were absent, we evaluated resection specimens for the presence or absence of an aganglionic segment. Available follow-up was obtained through patient chart analyses. In addition, chart review was performed to evaluate the patients that underwent frozen sections.

\section{Immunohistochemistry}

Immunohistochemistry was performed after xylene deparaffinization of replicate sections and utilization of the standard avidin-biotin complex method. Antibodies against the following antigens were used: peripherin (PJM50 monoclonal; 1:100; Vector Laboratories, Burlingame, CA, USA) and S-100 (polyclonal; 1:6000; Dako Corporation, Carpenteria, CA, USA). Antigen retrieval for peripherin immunohistochemistry included pressure cooker heat with target retrieval of $\mathrm{pH}$ 6.0. No pretreatment was used for the S-100 immunohistochemistry.

Peripherin immunostaining was considered positive if characteristic ganglion cell cytoplasmic staining was identified within a neural plexus (Figures 1a and b). The maximum diameter of submucosal nerve fibers was evaluated on S-100 immunostaining using visual micrometry. In cases positive for ganglion cells, care was taken not to measure bulbous widening of the nerve fibers as they coursed through the ganglia (Figure 1d).

\section{Frozen Section Preparation}

Tissues received for frozen section were cut into $5 \mu \mathrm{m}$ sections per routine protocol. One slide was fixed in $95 \%$ ethanol and stained with H\&E. A second slide was allowed to air dry and stained with the standard Diff Quick protocol.

\section{Results}

\section{Clinical Features}

A total of 138 consecutive cases were selected for evaluation. Two cases were non-diagnostic because of absence of submucosal tissue and repeat biopsies were not performed. Of the remaining cases, 90 were male and 46 were female. One hundred and nine 


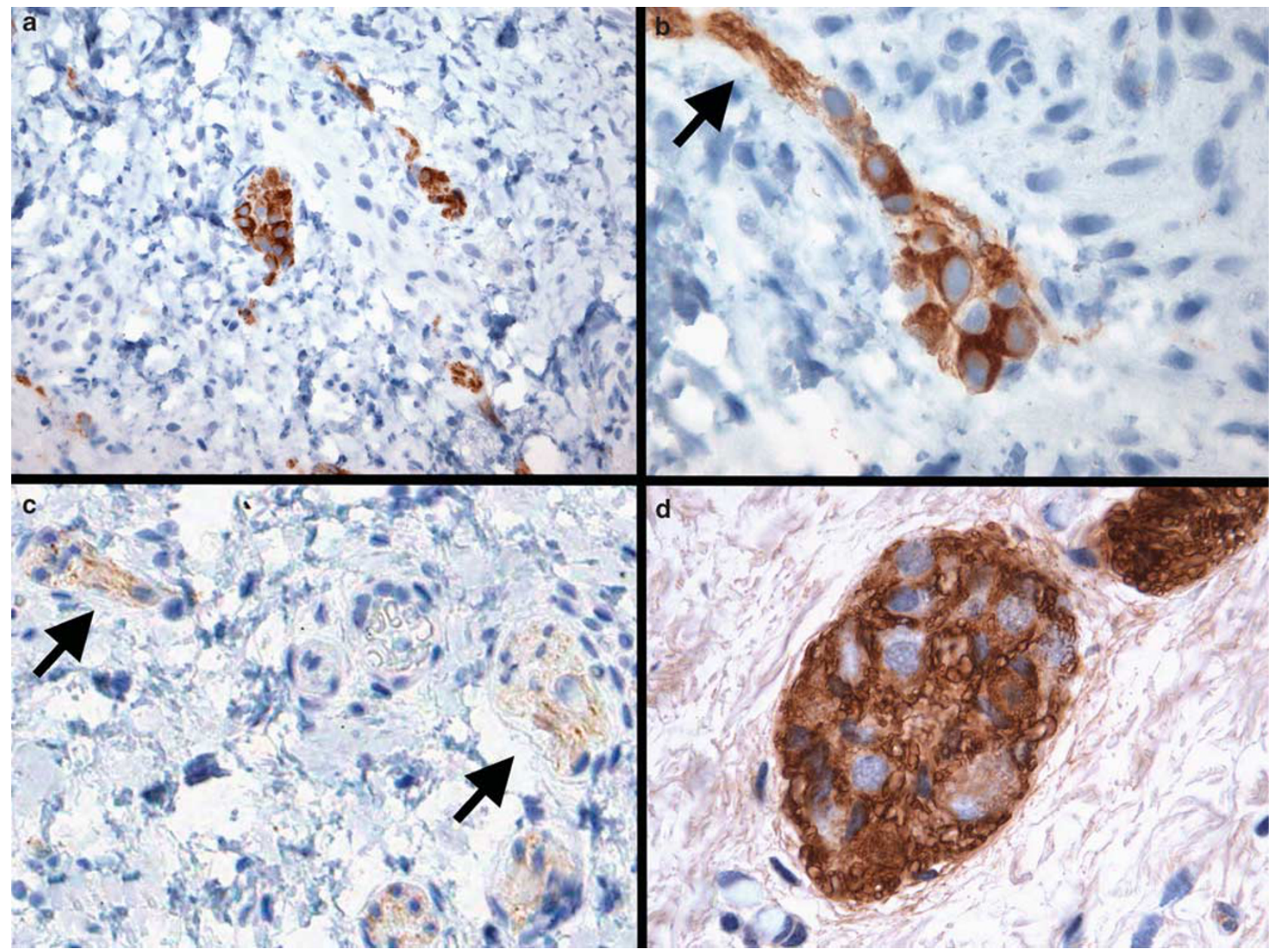

Figure 1 (a) Typical peripherin immunostaining of clusters of ganglion cells $(\times 40)$. (b) Peripherin staining of ganglion cells with granular deposits in a nerve bundle (arrow) as it enters the ganglion $(\times 100)$. (c) Faint linear and granular peripherin immunostaining of small nerve bundles within the submucosa (arrows) $(\times 40)$. (d) Negative image staining of ganglion cells in a non-Hirschsprung patient on S-100 immunostaining $(\times 100)$.

patients showed ganglion cells, and of these 66 were male and 43 were female. Of the 27 patients without ganglion cells, 23 were male and 4 were female, consistent with the male predominance of Hirschsprung disease.

Patients ranged in age from 2 days to 55 years. Forty-one $(41 \%)$ of the patients with ganglion cells presented at $<1$ month of age, $51 \%$ were 1 month to 1 year old and $8 \%$ were greater than 1 year old at presentation. Twenty-four (89\%) of the patients with Hirschsprung disease presented at or $<21$ days of age. The other patients presented at 3,7 , and 11 months.

All patients received rectal biopsies for evaluation of the presence of ganglion cells. Additional presenting symptoms in patients with ganglion cells were constipation ( $42 \%$ ), abdominal distention $(19 \%)$, meconium plug syndrome $(16 \%)$, short left colon syndrome $(10 \%)$, bowel obstruction $(5 \%)$, intestinal atresia $(3 \%)$, imperforate anus $(2 \%)$, bowel perforation $(2 \%)$, necrotizing enterocolitis $(1 \%)$, and sigmoid volvulus (1\%). Additional presenting symptoms in patients with Hirschsprung disease included abdominal distention (50\%), constipation $(20 \%)$, meconium plug syndrome $(15 \%)$, obstruction (5\%), intestinal atresia (5\%), and vomiting (5\%).

\section{Peripherin Immunohistochemistry}

Peripherin immunohistochemical staining showed a robust cytoplasmic staining within submucosal rectal ganglion cells (Figures 1a and b). We observed universal staining of ganglion cells in addition to a fine linear granular staining within nerve fibrils (Figures $1 \mathrm{~b}$ and $\mathrm{c}$ ).

\section{Patient Cohort}

Ganglion cells were identified in 109 patients (80\%) and $101(93 \%)$ of these were diagnosed on initial protocol, within a $24 \mathrm{~h}$ period. Eight $(7 \%)$ patients required repeat biopsies. One patient was initially non-diagnostic and repeat biopsy revealed ganglion cells. Two patients were initially negative on full 
protocol, but harbored low columnar or transitional mucosa, which was felt to be from the physiologic aganglionic segment. Repeat biopsies on both of these patients were positive for ganglion cells. Three patients had low numbers of ganglion cells on initial biopsy, ${ }^{1-3}$ and, at the request of the surgeons, had repeat biopsies with ganglion cells. One patient presented with a cecal perforation and ganglion cells were identified in the appendix and cecum. Follow-up rectal biopsy was positive for ganglion cells. One patient underwent resection of an atretic distal colon with confirmation of ganglion cells in the resected segment. Follow-up rectal biopsy was positive for ganglion cells.

Immunohistochemical stains for S-100 were available for evaluation in 107 patients with ganglion cells (mean diameter $20 \mu \mathrm{m}$ (range $8-55 \mu \mathrm{m}$ )). Of these, 103 harbored nerve bundles $<40 \mu \mathrm{m}$. Neural hypertrophy was identified in four $(4 \%)$ of these cases. Three of these cases (one male and two females) presented with constipation at the age of 4 days, 1 month, and 3 months, respectively. The corresponding maximum nerve diameters for these three cases were 48,40 , and $40 \mu \mathrm{m}$. The fourth patient was a 7-month-old male with imperforate anus and a maximum nerve diameter of $55 \mu \mathrm{m}$.

Follow-up was available on $49 \%$ of patients reported with ganglion cells. Two patients were further evaluated at other institutions for persistent severe constipation. At these institutions, the presence of ganglion cells was confirmed. No other pertinent adverse follow-up was identified.

Ganglion cells were absent in 27 patients $(20 \%)$. Before formal resection, 22 of these patients underwent repeat biopsies in the operating room to define the extent of aganglionosis. The repeat biopsies confirmed an aganglionic segment in all of these cases.

On the original suction biopsy of these Hirschsprung disease cases, neural hypertrophy (Figures 2a and b) was identified in 22 patients (81\% (mean $54 \mu \mathrm{m}$, diameter (range $40-150 \mu \mathrm{m}$ ))). The remaining patients showed nerve fibers that measured 33, 33, 20, 18, and $15 \mu \mathrm{m}$ in maximum diameter. Twenty-six patients underwent Duhamel procedure with confirmed aganglionic segment (mean length $10.8 \mathrm{~cm}$ (length range $4.0-26.2 \mathrm{~cm}$ )). All patients had bowel movements postoperatively. One of these patients had postoperative anal stenosis that responded to dilations. The final patient had severe congenital abnormalities, and colostomy only was performed. This patient had intraoperative confirmation of aganglionosis.

\section{Frozen Sections}

Frozen section analyses were performed on 26 patients. Of these 26, 22 patients had resections for Hirschsprung disease, two had repeat biopsies (intraoperative confirmation of the presence of ganglion cells), and two had de novo biopsies. The Diff Quick protocol stained the cytoplasm of ganglion cells bright blue, expediting the intraoperative evaluations (Figures 2c and d). A total of 57 frozen sections were performed on these patients, with a $95 \%$ (54 of 57) concordance with permanent sections. The three outliers were determined to be sampling errors.

\section{Discussion}

Evaluation of suction rectal biopsies for the presence of ganglion cells remains very challenging, especially in negative or equivocal cases. The majority of these biopsies are performed on newborn infants, in which the immature ganglion cells can be confused with endothelial cells or fibroblasts. As a result of these difficulties, a large number of histochemical and immunohistochemical stains have been proposed to assist in the identification of ganglion cells, or to delineate the nature of the nerve fibers in suction rectal biopsies. In 1988, Robey et $a l^{6}$ used NSE to decorate ganglion cells and had a 91\% confirmatory rate. In the mid-1990s, peripherin ${ }^{7}$ and cathepsin $\mathrm{D}^{11}$ immunostains were introduced, also with good success. Thereafter, multiple series of staining protocols were reported with antibodies against bcl-2, ${ }^{12}$ NeuN,${ }^{13}$ BMPRIA, ${ }^{14}$ neurofilament, ${ }^{7,15}$ and tubulin, ${ }^{7}$ all with good results. In 2006, Karim et $a l^{16}$ identified wild-type ret oncoprotein within ganglion cells and concluded that ganglion cells were easier to identify by staining with a ret oncoprotein immunostain than with H\&E staining alone. Most recently, Burtelow and Longacre, ${ }^{17}$ reported on the utility of MAP-2 immunostaining of ganglion cells. They were successful in routinely identifying ganglion cells on immunohistochemical staining in equivocal cases.

Historically, the evaluation of submucosal nerve trunks and muscularis mucosa and lamina propria nerve fibrils have been of utmost importance in confirming patients with suspected aganglionosis. Acetylcholinesterase histochemical staining has been extensively used as an adjunct to identifying and typifying parasympathetic nerves in rectal biopsies. The procedure, which requires acquisition of frozen tissue, is potentially toxic, and is also time consuming. In addition, the stains may be difficult to interpret, particularly in ambiguous cases. ${ }^{3,4}$ The demands of interpretation of this stain are well documented with false positive, ${ }^{4-6,8,9}$ false negative ${ }^{18-22}$ and equivocal ${ }^{19,22}$ results.

Owing to the diagnostic limitations of acetylcholinesterase histochemical staining, a number of immunohistochemical stains have been used to further define the nature of nerves within suction rectal biopsies. S-100 immunostaining in this setting was first introduced by Robey et al in $1988 .^{6}$ It consistently stained submucosal nerves and negatively highlighted ganglion cells in ganglia. 


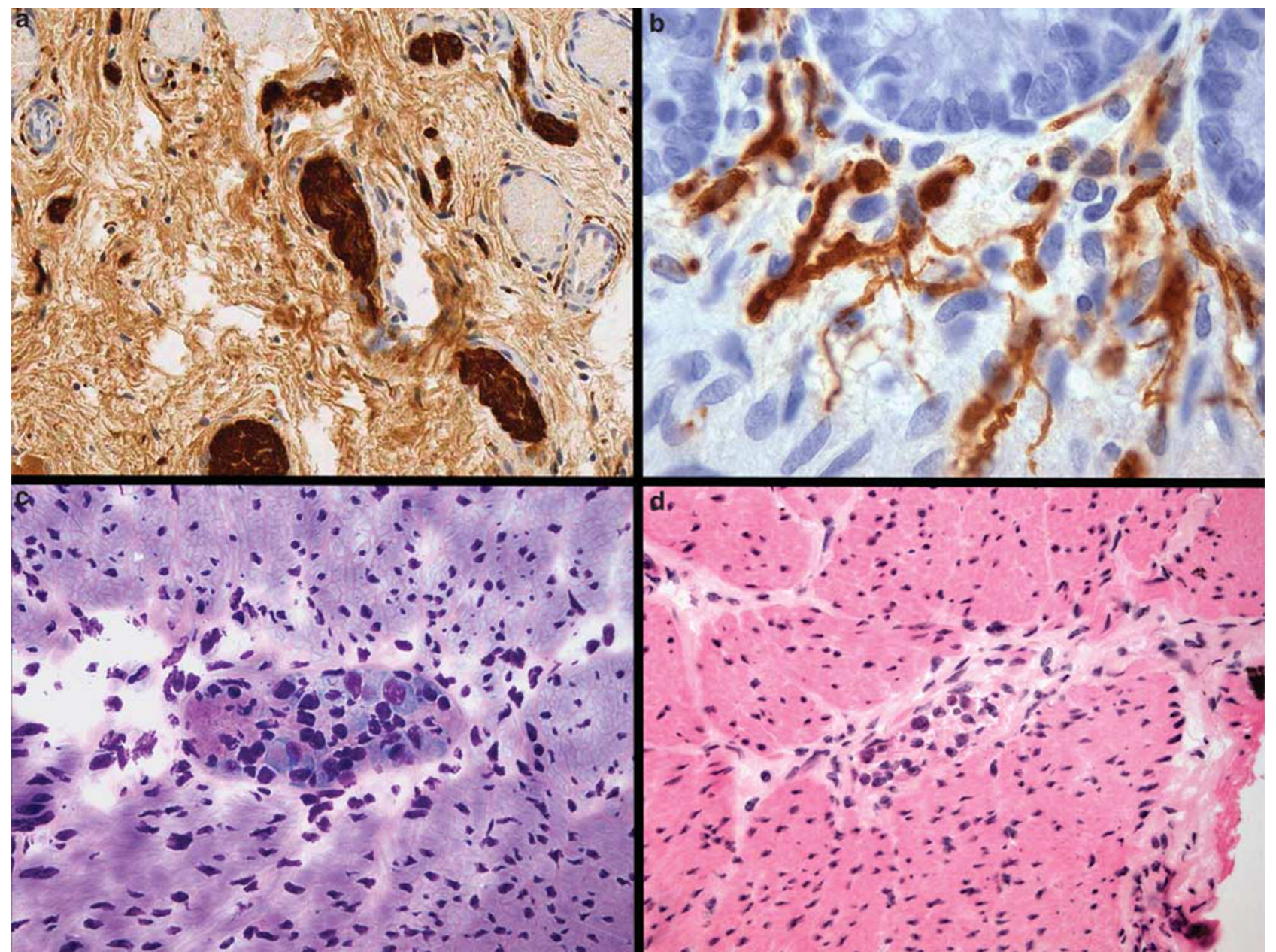

Figure 2 (a) S-100 immunostaining highlights neural hypertrophy in a patient with Hirschsprung disease $(\times 40)$. (b) Increased muscularis mucosae and lamina propria nerve fibrils in a patient with Hirschsprung disease on S-100 staining $(\times 100)$. (c) Diff Quik staining reveals bright blue cytoplasm of ganglion cells $(\times 40)$. (d) H\&E control of frozen section $(\times 20)$.

A decade later, Monforte-Muñoz et $a l^{5}$ studied selected S-100 immunostaining on 20 Hirschsprung patients and 50 non-Hirschsprung patients. They found that $90 \%$ of their Hirschsprung cases harbored nerve bundles of $\geqslant 40 \mu \mathrm{m}$. They concluded that S-100 immunostaining represented a useful ancillary modality in cases without demonstrable ganglion cells. In addition, antibodies to MAP- $5,{ }^{4}$ GLUT- $1,{ }^{23}$ and GFAP ${ }^{24}$ have been used to delineate submucosal nerves in rectal biopsies. Of interest, recently Barshack et $a l^{25}$ reported that calretinin immunostaining was absent in intrinsic nerve fibers in Hirschsprung colons, whereas it is identified in ganglion cells and intrinsic nerve fibers of nonHirschsprung patients. As a follow-up, Kapur et al ${ }^{22}$ retrospectively applied calretinin immunostain to 14 Hirschsprung and 17 non-Hirschsprung patients, with staining results similar to the findings of Barshack et al. ${ }^{25}$ Further, this group compared the calretinin immunostain with the acetylcholinesterase stains performed at initial biopsy. They concluded that the calretinin immunohistochemical stain was superior to acetylcholinesterase histo- chemical stain in these evaluations. There were fewer major discrepancies and fewer equivocal interpretations. In addition, Guinard-Samuel et $a l^{26}$ immunostained 131 cases of suspected Hirschsprung disease with calretinin, and also concluded that this immunohistochemical stain was superior to acetylcholinesterase in this clinical scenario.

The rapid intraoperative identification of ganglion cells has a significant function in the management of these patients. It shortens operative time, directs the type and extent of surgical resection, and improves postoperative outcomes. Although H\&E staining remains the gold standard method for identifying ganglion cells, intraoperative frozen section $\mathrm{H} \& \mathrm{E}$ analysis can be fraught with limitations. In this setting, ganglion cells stain a pale pink color as do hypertrophic nerve fibers and smooth muscle cells. In addition, the majority of Hirschsprung patients are infants with small, immature ganglia that are few in number. As a result, these cells can be easily overlooked on routine frozen sections. Consequently, numerous immunohistochemical stains have been evaluated to facilitate and improve 
accuracy at the time of frozen section. These stains include neurofilament, tubulin, synaptophysin, peripherin, NCAM/CD56, and acetylcholinesterase. ${ }^{15,27,28}$ Beschorner et $a l^{27}$ reported that neurofilament, synaptophysin, peripherin, NCAM/CD56, and acetylcholinesterase were not helpful in identifying ganglion cells at the time of frozen section. Further, in 2007, a Canadian group published positive results with acetone fixation and rapid immunofluorescence staining with antibodies against tubulin and neurofilament 68 on frozen tissue. ${ }^{15}$ This procedure, however, seems to be labor intensive. Recently, a less toxic version of the acetylcholinesterase stain has shown promising results as a marker of ganglion cells in frozen tissue. ${ }^{21}$ As stated above, however, the interpretation may be difficult, particularly with a frozen preparation.

We have found that the use of the Diff Quik stain on frozen tissue highlights ganglion cells by staining their cytoplasm a bright blue. The stain is readily available, inexpensive, and non-toxic, and can be applied directly to frozen tissue sections. In addition, it requires no technical expertise to perform. The contrast between the bright blue staining in the ganglion cells and the pale purple staining in the remainder of the tissues facilitates fast and easy identification of the ganglion cells in the intraoperative setting. When used in concert with $\mathrm{H} \& \mathrm{E}-$ stained slides, the Diff Quik stain acts as a complement, and thus shortens frozen section interpretation times.

In conclusion, close examination of multiple H\&E sections coupled with the adjuvant immunohistochemical stains of peripherin and S-100 proved to be a highly sensitive and specific modality for the identification of ganglion cells in suction rectal biopsies. Over the course of the study, this diagnostic accuracy was maintained among several nongastrointestinal and non-pediatric pathologists. This protocol improved laboratory efficiency, as serial $\mathrm{H} \& \mathrm{E}$ sections were needed in fewer than $30 \%$ of cases. In addition, routine obtaining of frozen tissue was not necessary. All of this was accomplished while maintaining a $24 \mathrm{~h}$ turnaround time in $93 \%$ of cases.

\section{Disclosure/conflict of interest}

The authors declare no conflict of interest.

\section{References}

1 van Heyningen V. Genetics. One gene-four syndromes. Nature 1994;367:319-320.

2 Uesaka T, Nagashimada M, Yonemura S, et al. Diminished Ret expression compromises neuronal survival in the colon and causes intestinal aganglionosis in mice. J Clin Invest 2008;118: 1890-1898.
3 Challa VR, Moran JR, Turner CS, et al. Histologic diagnosis of Hirschsprung's disease. Am J Clin Pathol 1987;88:324-328.

4 Tam PKH, Owen G. An immunohistochemical study of neuronal microtubule-associated proteins in Hirschsprung's disease. Hum Pathol 1993;24: 424-431.

5 Monforte-Munoz H, Gonzales-Gomez I, Rowland JM, et al. Increased submucosal nerve trunk caliber in aganglionosis: a 'positive' and objective finding in suction biopsies and segmental resections in Hirschsprung's disease. Arch Pathol Lab Med 1998;122: 721-725.

6 Robey SS, Kuhajda FP, Yardley JH. Immunoperoxidase stains of ganglion cells and abnormal mucosal nerve proliferations in Hirschsprung disease. Hum Pathol 1988;19:432-437.

7 Szabolcs MJ, Visser J, Shelanski ML, et al. Peripherin: a novel marker for the immunohistochemical study of malformations of the enteric nervous system. Pediatr Pathol Lab Med 1996;16:51-70.

8 Petchasuwan C, Pintong J. Immunohistochemistry for intestinal ganglion cells and nerve fibers: aid in the diagnosis of Hirschsprung's disease. J Med Assoc Thai 2000;83:1402-1409.

9 Piotrowska AP, Solari V, Puri P. Distribution of interstitial cells of Cajal in the internal anal sphincter of patients with internal anal sphincter achalasia and Hirschsprung disease. Arch Pathol Lab Med 2003;127:1192-1195.

10 Matsuda H, Hirato J, Kuroiwa M, et al. Histopathological and immunohistochemical study of the enteric innervations among various types of aganglionoses including isolated and syndromic Hirschsprung disease. Neuropathology 2006;26:8-23.

11 Abu-Alfa AK, Kuan SF, West AB, et al. Cathepsin D in intestinal ganglion cells. A potential aid to diagnosis in suspected Hirschsprung's disease. Am J Surg Pathol 1997;21:201-255.

12 Wester T, Olsson Y, Olsen L. Expression of bcl-2 in enteric neurons in normal bowel and Hirschsprung disease. Arch Pathol Lab Med 1999;123:1264-1268.

13 Yang S, Donner LR. Detection of ganglion cells in the colonic plexuses by immunostaining for neuronspecific marker NeuN: an aid for the diagnosis of Hirschsprung disease. Appl Immunohistochem Mol Morphol 2002;10:218-220.

14 Brewer KC, Mwizerva O, Goldstein AM. BMPRIA is a promising marker for evaluating ganglion cells in the enteric nervous system: a pilot study. Hum Pathol 2005;36:1120-1126.

15 Staines WA, Bettolli M, De Carli C, et al. Fast evaluation of intraoperative biopsies for ganglia in Hirschsprung's disease. J Pediatr Surg 2007;42: 2067-2070.

16 Karim S, Hession C, Marconi S, et al. The identification of ganglion cells in Hirschsprung disease by the immunohistochemical detection of ret oncoprotein. Am J Clin Pathol 2006;126:49-54.

17 Burtelow MA, Longacre TA. Utility of microtubule associated protein-2 (MAP-2) immunohistochemistry for identification of ganglion cells in paraffin-embedded rectal suction biopsies. Am J Surg Pathol 2009; 33:1025-1030.

18 Ariel I, Vinograd I, Lernau OZ, et al. Rectal mucosal biopsy in aganglionosis and allied conditions. Hum Pathol 1983;14:991-995. 
19 Athow AC, Filipe MI, Drake DP. Problems and advantages of acetylcholinesterase histochemistry of rectal suction biopsies in the diagnosis of Hirschsprung's disease. J Pediatr Surg 1990;25:520-526.

20 Sun C-CJ, Caniano DA, Hill JL. Intestinal aganglionosis. A histologic and acetylcholinesterase histochemical study. Pediatr Pathol 1987;7:421-435.

21 Huntley CC, Shaffner LD, Challa VR, et al. Histochemical diagnosis of Hirschsprung disease. Pediatrics 1982;69:755-761.

22 Kapur RP, Reed RC, Finn LS, et al. Calretinin immunohistochemistry versus acetylcholinesterase histochemistry in the evaluation of suction rectal biopsies for Hirschsprung disease. Pediatr Dev Pathol 2009; 12:6-15.

23 Kakita Y, Oshiro K, O’Briain DS, et al. Selective demonstration of mural nerves in ganglionic and aganglionic colon by immunohistochemistry for glucose transporter-1: prominent extrinsic nerve pattern staining in Hirschsprung disease. Arch Pathol Lab Med 2000;124:1314-1319.

24 Kawana T, Nada O, Ikeda S, et al. Distribution and localization of glial fibrillary acidic protein in colons affected by Hirschsprung's disease. J Pediatr Surg 1989;24:448-452.

25 Barshack I, Fridman E, Goldberg I, et al. The loss of calretinin expression indicates aganglionosis in Hirschsprung's disease. J Clin Pathol 2004;57:712-716.

26 Guinard-Samuel V, Bonnard A, De Lagausie P, et al. Calretinin immunohistochemistry: a simple and efficient tool to diagnose Hirschsprung disease. Mod Pathol 2009;22:1379-1384.

27 Beschorner R, Mittelbronn M, Bekure $\mathrm{K}$, et al. Problems in fast intraoperative diagnosis in Hirschsprung's disease. Folia Neuropathol 2004;42:191-195.

28 Kobayashi H, Miyahara K, Kusafuka J, et al. A new rapid acetylcholinesterase staining kit for diagnosing Hirschsprung's disease. Pediatr Surg Int 2007;23:505-508. 